\title{
Theoretical $\mathrm{H}_{2} \mathrm{CO}$ emission from protostellar envelopes
}

\author{
C. Ceccarelli ${ }^{1}$, S. Maret ${ }^{2}$, A. G. G. M. Tielens ${ }^{3}$, A. Castets $^{4}$, and E. Caux ${ }^{2}$ \\ ${ }^{1}$ Laboratoire d'Astrophysique, Observatoire de Grenoble, BP 53, 38041 Grenoble cedex 09, France \\ 2 CESR CNRS-UPS, BP 4346, 31028 Toulouse Cedex 04, France \\ 3 SRON, PO Box 800, 9700 AV Groningen, The Netherlands \\ ${ }^{4}$ Observatoire de Bordeaux, BP 89, 33270 Floirac, France
}

Received 22 May 2003 / Accepted 30 July 2003

\begin{abstract}
We present theoretical predictions of the formaldehyde emission from the envelopes of low mass protostars. The model accounts for the density profile across the envelope, assuming the Shu (1977) solution for the collapse, as well as for the gas temperature profile. The gas temperature is derived from the thermal balance according to the model previously developed by Ceccarelli et al. (1996). The formaldehyde abundance profile is approximated by a step function: in the outer envelope it is similar to that of molecular clouds, whereas in the inner envelope, where the dust temperature reaches the ice mantle evaporation temperature, it jumps to larger values. The results of the modeling for a $30 L_{\odot}$ source are reported for a large range of values of the formaldehyde abundance, both in the inner and outer envelope. Additional results for sources with different luminosities can be found in the web site www-laog.obs.ujf-grenoble.fr/ ceccarel/mepew/mepew.html. They are meant to be directly used by the interested reader to estimate the two main parameters of the model: the inner and outer formaldehyde abundance. The model is applied to the observations of the well known low mass protostar IRAS 16293-2422 to illustrate the practical use of the diagnostic tools. Furthermore, we discuss how the observation of the $\mathrm{H}_{2}^{12} \mathrm{CO}$ and $\mathrm{H}_{2}^{13} \mathrm{CO}$ lines can in principle be used to discriminate between infalling and outflowing gas. Finally, the same web site hosts simpler non-LTE LVG predictions for a large range of densities, temperatures and column densities of several molecules. Again the goal of the web site is to provide users with easy to use theoretical predictions for a first assessement of expected and/or observed signals.
\end{abstract}

Key words. ISM: abundances - ISM: molecules - stars: formation

\section{Introduction}

That low-mass stars form from the collapse of dense fragments of molecular clouds is a widely accepted statement. Despite the large acceptance of this statement, yet the observational evidence of the actual collapse in low-mass protostars is claimed (and debated) in only a handful of objects. The reason is the difficulty to detect unambigously infall motions. So far the main means to probe the infall is via the study of the line profile of optically thick molecular lines. The basic idea consists in using optically thick lines which show self-absorption: in a collapsing envelope such lines are predicted to have the blueshifted peak less attenuated and therefore brighter than the redshifted peak (Leung \& Brown 1977; Adelson \& Leung 1988; Zhou 1992; Walker et al. 1994). Lately, a number of surveys have been carried out which exploit this method (Gregersen et al. 1997; Mardones et al. 1997; Williams \& Myers 1999; Choi 2002; see Evans 1999 for a review). The main result from these surveys is the identification of a few "collapsing" candidates, which further studies are expected to confirm as such. An

Send offprint requests to: C. Ceccarelli, e-mail: ceccarel@obs.ujf-grenoble.fr outstanding example is the work by Di Francesco et al. (2001), who detected the inverse PCygni profile of a formaldehyde line with interferometric observations towards the low mass binary protostar NGC 1333-IRAS 4.

Recently, a different, complementary method based on the study of the line fluxes rather than on the line profiles has been proposed (Ceccarelli et al. 1996, CHT96 hereinafter; Ceccarelli et al. 2000; Maret et al. 2002). The basic idea is that specific lines from selected molecules are capable to probe different regions of the envelope surrounding a protostar. If the envelope is collapsing, it has a well defined thermal and chemical structure, which in turn implies a well defined line flux spectrum. Ceccarelli et al. (2000) and Maret et al. (2002) considered the lines from the major coolants of the gas of the envelope, namely the observed $\mathrm{O}^{0}$ and $\mathrm{H}_{2} \mathrm{O}$ FIR lines, and applied the method to two well studied protostars, IRAS 16293-2422 and NGC 1333-IRAS 4, where detection of infall has been claimed by other authors (Walker et al. 1986; Zhou 1995; Narayanan et al. 1998; Di Francesco et al. 2001; Choi 2001). Encouraging enough, both methods, the one based on line profiles and the one based on line fluxes, give similar results in the derived physical conditions such as the mass accretion rate and the 
mass of the central object. Hence, although the ultimate prove of the collapse clearly needs also the line profile "certification", the "line fluxes" method can be very useful to identify collapsing envelopes in protostars and suggests itself another hallmark of a collapsing envelope.

Unfortunately, because $\mathrm{O}^{0}$ and $\mathrm{H}_{2} \mathrm{O}$ FIR lines are not easily detectable, as they lie in a wavelength range obscured by the terrestrial atmosphere, the method based on the main gas coolants has not been used on a large number of objects. So far, this method has been applied only to the two above mentioned protostars. The HERSCHEL mission with two superb spectrometers on board - HIFI and PACS - is expected to change this in 2007. In this article, we pursue the basic idea that the line fluxes of appropriate molecules possess the hallmark of the collapse, and we apply the method to the formaldehyde. The reason for using $\mathrm{H}_{2} \mathrm{CO}$ lines is evident: $\mathrm{H}_{2} \mathrm{CO}$ lines, with a variety of excitation energies, are readly observable in ground-based windows. Furthermore, $\mathrm{H}_{2} \mathrm{CO}$ is one of the most abundant species in ices after $\mathrm{H}_{2} \mathrm{O}$ in massive protostars (e.g. van Dishoeck \& Blake 1998; Gibb et al. 2000), as well as in the Hot Cores around low mass protostars (Ceccarelli et al. 2000; Schöier et al. 2002), where complex molecules, typical of massive Hot Cores, have also been detected (Cazaux et al. 2003). In this paper we describe the theoretical modeling of the ortho $\mathrm{H}_{2} \mathrm{CO}$ lines and how selected $\mathrm{H}_{2}^{12} \mathrm{CO}$ and $\mathrm{H}_{2}^{13} \mathrm{CO}$ lines can be used to constrain the key parameters of the model, namely the mass of the central object, the accretion rate and the $\mathrm{H}_{2} \mathrm{CO}$ abundance. In addition they allow to discriminate between infalling and outflowing gas. The model has been already succesfully applied to interprete the observed $\mathrm{H}_{2} \mathrm{CO}$ line emission towards IRAS 16293-2422 (Ceccarelli et al. 2000, 2001), giving predictions recently fully confirmed by the independent analysis of Schöier et al. (2002). The bottom line of the proposed method is the capacity of the $\mathrm{H}_{2} \mathrm{CO}$ lines to infer the temperature and density of the gas (e.g. Mangum et al. 1990; Mangum \& Wootten 1993; Mangum et al. 1999) in different regions of the envelope and therefore to be able to probe the structure of the envelope itself.

This article is organized as follows. After a description of the model (Sect. 2), we report plots relative to a $30 L_{\odot}$ source (Sect. 3). In Sect. 4 we discuss diagnostic plots and apply them to the case of the protostar IRAS 16293-2224. Results for differents source luminosities are reported in the web site www-laog.obs.ujf-grenoble.fr/ ceccarel/ mepew/mepew.html. We briefly describe the content of the site (Sect. 5), which also hosts computations of non-LTE LVG model, for several molecules.

\section{The model description}

\subsection{Physical structure of the envelope}

The physical and thermal structure of a collapsing envelope is derived from the model developed in CHT96. The envelope density structure and dynamics follows the "inside-out" collapse solution developed by Shu (1977), for a spherical initial isothermal sphere undergoing collapse. In the outer envelope, not affected by the collapse yet, the molecular hydrogen number density distribution $n_{\mathrm{H}_{2}}(r)$ is given by:

$$
\begin{aligned}
n_{\mathrm{H}_{2}}(r) & =\frac{a^{2}}{2 \pi \mu m_{\mathrm{H}} G} r^{-2} \\
& =2.8 \times 10^{8}\left(\frac{a}{0.35 \mathrm{~km} \mathrm{~s}^{-1}}\right)^{2} r_{100 \mathrm{AU}}^{-2} \mathrm{~cm}^{-3}
\end{aligned}
$$

where $a$ is the sound speed, $m_{\mathrm{H}}$ is the hydrogen mass, $\mu$ is the mean molecular mass in amu units, equal to $2.8, r_{100 \mathrm{AU}}$ is the distance from the center in $100 \mathrm{AU}$ units, and $G$ is the gravitational constant. In the inner collapsing regions the density is described by the free-fall solution:

$$
\begin{aligned}
n_{\mathrm{H}_{2}}(r) & =\frac{1}{4 \pi \mu m_{\mathrm{H}}}\left(\frac{\dot{M}^{2}}{2 G M_{\star}}\right)^{\frac{1}{2}} r^{-\frac{3}{2}} \\
& =1.2 \times 10^{7}\left(\frac{\dot{M}_{-5}^{2}}{M_{\star} r_{100 \mathrm{AU}}^{3}}\right)^{\frac{1}{2}} \mathrm{~cm}^{-3} .
\end{aligned}
$$

The free fall velocity is given:

$$
v(r)=\left(\frac{2 G M_{\star}}{r}\right)^{\frac{1}{2}}=4.2\left(\frac{M_{\star 1}}{r_{100 \mathrm{AU}}}\right)^{\frac{1}{2}} \mathrm{~km} \mathrm{~s}^{-1},
$$

where $\dot{M}$ is the mass accretion rate, related to the sound speed by

$$
\dot{M}=0.975 \frac{a^{3}}{G}=10^{-5}\left(\frac{a}{0.35 \mathrm{~km} \mathrm{~s}^{-1}}\right)^{3} M_{\odot} \mathrm{yr}^{-1} .
$$

$\dot{M}_{-5}$ is $\dot{M}$ in units of $10^{-5} M_{\odot} \mathrm{yr}^{-1}$, and $M_{\star 1}$ is the mass of the central object $M_{\star}$ in units of $1 M_{\odot}$. The spherical symmetry is conserved through the collapse in this model. In this sense, the model gives accurate results only for that part of the envelope in which the spherical symmetry is a good approximation, i.e. probably for radii larger than a few tens of AUs (Ceccarelli et al. 2000a). At smaller scales large deviations from the spherical symmetry are expected because of the presence of circumstellar disks.

The CHT96 model computes self-consistently the chemical abundance of the main gas coolants of the envelope, namely the atomic oxygen, the water and $\mathrm{CO}$ molecules, and the thermal balance, taking the line cooling and radiative transfer aspects fully into account. The molecular abundances are followed during the evolution of the protostar. Whereas the $\mathrm{CO}$ abundance is constant across the envelope and during the collapse, the $\mathrm{H}_{2} \mathrm{O}$ abundance jumps in the region where the dust temperature exceeds $100 \mathrm{~K}$, reflecting the thermal evaporation of the icy grain mantles. Closer to the central object, when the gas temperature reaches about $250 \mathrm{~K}$, the $\mathrm{H}_{2} \mathrm{O}$ abundance has a second jump caused by the conversion of atomic oxygen into water via endothermic gas phase reactions. In the same region the atomic oxygen abundance drops to zero, following this conversion. The thermal structure is governed by the heating due to compression of the gas and water absorption of the NIR photons emitted by the warm dust, and by the cooling via rotational line emission of $\mathrm{CO}$ in the outer part of the envelope $(\geq 1000 \mathrm{AU})$, of $\mathrm{H}_{2} \mathrm{O}$ in the inner regions $(\leq 200 \mathrm{AU})$ and of $\mathrm{O}^{0}$, $\mathrm{CO}$ and $\mathrm{H}_{2} \mathrm{O}$ in the intermediate parts. The water, atomic oxygen and $\mathrm{CO}$ abundance are therefore "hidden" parameters of 
the model, controlled in essence by the oxygen and carbon elemental abundances. In the present study, we use the values derived by the above mentioned studies of IRAS 16293-2422 and NGC 1333-IRAS 4 (Ceccarelli et al. 2000; Maret et al. 2002), namely:

$2.5 \times 10^{-4}$ for atomic oxygen;

$1 \times 10^{-4}$ for $\mathrm{CO}$;

$5 \times 10^{-7}$ for the water abundance in the cold part of the envelope;

$5 \times 10^{-6}$ for the water abundance injected in the gas phase when the grain mantles evaporate at $100 \mathrm{~K}$.

For a thorough discussion on the dependence of the gas temperature profile on the choice of the latter three parameters, we refer the interested reader to Maret et al. (2002). The central mass of the protostar $M_{\star}$ and the mass accretion rate $\dot{M}$ are the two parameters of the model which govern directly the overall density and velocity structure, and, indirectly, both the chemical and thermal structure. They are the two key parameters that the present study addresses. We varied the central mass between 0.5 and $1.5 M_{\odot}$ and the mass accretion rate between 0.5 and $5 \times 10^{-5} M_{\odot} \mathrm{yr}^{-1}$. Note that the age of the protostar in this model is given by the central mass divided by the mass accretion rate, which is assumed to be constant.

\section{2. $\mathrm{H}_{2} \mathrm{CO}$ line emission}

The $\mathrm{H}_{2} \mathrm{CO}$ distribution across the envelope directly influences the $\mathrm{H}_{2} \mathrm{CO}$ line emission. In this study, we approximate the formaldehyde abundance profile with a step function. In the outer envelope the $\mathrm{H}_{2} \mathrm{CO}$ abundance, $x_{\text {cold }}$, is relatively low and similar to that observed in molecular clouds. In the innermost regions of the envelope, where the dust reaches the evaporation temperature of the polar ices, $100 \mathrm{~K}$ (Tielens \& Allamandola 1987), the $\mathrm{H}_{2} \mathrm{CO}$ abundance, $x_{\text {warm }}$, jumps to larger values. A similar step function model has been previously used for modeling the $\mathrm{H}_{2} \mathrm{CO}$ line spectrum of the well studied source IRAS 16293-2422 (Ceccarelli et al. 2000; Schöier et al. 2002), a sample of low mass protostars (Maret et al. in preparation), and some massive protostars (van der Tak et al. 2000). Indeed, a detailed study of the $\mathrm{H}_{2} \mathrm{CO}$ line emission and its spatial distribution in IRAS $16293-2422$ shows that the $\mathrm{H}_{2} \mathrm{CO}$ abundance is $4 \times 10^{-10}$ in the region where the dust temperature is lower than about $50 \mathrm{~K}$, it jumps to $4 \times 10^{-9}$ when the dust temperature reaches $50 \mathrm{~K}$, and it jumps to $1 \times 10^{-8}$ when the dust is warmer than $100 \mathrm{~K}$ (Ceccarelli et al. 2001). The intermediate jump at $50 \mathrm{~K}$ can reflect the evaporation of pure $\mathrm{H}_{2} \mathrm{CO}$ ices. Nevertheless, in order to minimize the number of free parameters, we choose to approximate the formaldehyde abundance profile with one single step function. This implies that $x_{\text {cold }}$ is likely an average abundance in the outer envelope. We varied $x_{\text {cold }}$ between $10^{-10}$ and $3 \times 10^{-8}$, and $x_{\text {warm }}$ between $10^{-7}$ and $3 \times 10^{-6}$. The luminosity of the protostar in the present study is fixed and it is set equal to $30 L_{\odot}$. All the line fluxes are computed for a source at a distance of $160 \mathrm{pc}$. The $\mathrm{H}_{2}^{12} \mathrm{CO} /$ $\mathrm{H}_{2}^{13} \mathrm{CO}$ ratio is assumed to be equal to 70 (e.g. Boogert et al. $2000)$, although the actual value is somewhat uncertain $(20 \%$; e.g. Wilson \& Rood 1994). We expect that the results from
Table 1. Values of the parameters adopted for the test case.

\begin{tabular}{cc}
\hline \hline Parameter & Value \\
\hline$M_{\star}$ & $0.8 M_{\odot}$ \\
$\dot{M}$ & $3 \times 10^{-5} M_{\odot} \mathrm{yr}^{-1}$ \\
$x_{\text {warm }}$ & $3 \times 10^{-7}$ \\
$x_{\text {cold }}$ & $3 \times 10^{-9}$ \\
\hline$x(\mathrm{CO})$ & $1 \times 10^{-4}$ \\
$x(\mathrm{O})$ & $2.5 \times 10^{-4}$ \\
$x_{\text {cold }}\left(\mathrm{H}_{2} \mathrm{O}\right)$ & $5 \times 10^{-7}$ \\
$x_{\text {warm }}\left(\mathrm{H}_{2} \mathrm{O}\right)$ & $5 \times 10^{-6}$ \\
\hline
\end{tabular}

the model are affected by about the same uncertainty. Finally, the model computes the level population of the first 40 levels of the ortho form of $\mathrm{H}_{2} \mathrm{CO}$. The molecular data are from the JPL catalogue (http://spec.jpl.nasa.gov/home.html). The collisional coefficients are from Green (1991), who computed them between 10 and $300 \mathrm{~K}$, i.e. in the range of the modeled envelope temperatures. Note that all abundances are reported here with respect to $\mathrm{H}_{2}$. Finally, we did not consider the para $\mathrm{H}_{2} \mathrm{CO}$ lines for the ortho to para ratio is poorly known and would hence be an additional parameter of the model.

\section{Model results}

We first report the computed $\mathrm{H}_{2} \mathrm{CO}$ line spectrum of a test case, to illustrate the general characteristics of the predicted $\mathrm{H}_{2} \mathrm{CO}$ spectrum. Then, in the following paragraphs we discuss thoroughly how the $\mathrm{H}_{2} \mathrm{CO}$ line spectrum varies with the four parameters of the model, namely the mass of the central object $M_{\star}$, the mass accretion rate $\dot{M}$, the $\mathrm{H}_{2} \mathrm{CO}$ abundance in the outer cold envelope $x_{\text {cold }}$, and in the inner warm envelope $x_{\text {warm }}$. Finally, a last paragraph discusses the differences between the $\mathrm{H}_{2} \mathrm{CO}$ emission due to the infalling envelope versus the emission due to the outflowing material.

\subsection{The test case $\mathrm{H}_{2} \mathrm{CO}$ line spectrum}

The parameters adopted in the test case are reported in Table 1. These values, derived for IRAS 16293-2422, are comparable, within one order of magnitude, to those derived for several observed Class 0 protostars (Maret et al. in preparation). In the following all fluxes are given in erg $\mathrm{s}^{-1} \mathrm{~cm}^{-2}$. Although the conversion in $\mathrm{K} \mathrm{km} \mathrm{s}^{-1}$ would have been more pratical for the observers, the signal in $\mathrm{K} \mathrm{km} \mathrm{s}^{-1}$ depends on the beam of the telescope used for the observation and how it matches the predicted extent of the emission. A description of a practical example, with the relevant conversions, is reported in Sect. 4.

Table 2 reports the predicted fluxes of the ortho $\mathrm{H}_{2} \mathrm{CO}$ lines for the test case, and Fig. 1 shows the relative spectrum. Lines from $K_{-1}=1$ and $K_{-1}=3$ ladders (the nomenclature for the level is $\left.J_{K_{-1}, K_{+1}}\right)$ are evident both in the $\mathrm{H}_{2}^{12} \mathrm{CO}$ and $\mathrm{H}_{2}^{13} \mathrm{CO}$ spectra, where the $K_{-1}=1$ transitions are the series with the brightest lines. The $\mathrm{H}_{2}^{12} \mathrm{CO}$ line spectrum shows a first peak at $E_{\text {up }} \sim 40 \mathrm{~cm}^{-1}$ which reflects the excitation conditions of the outer envelope. This is because the $\mathrm{H}_{2}^{12} \mathrm{CO}$ lines with 
Table 2. Ortho $\mathrm{H}_{2}^{12} \mathrm{CO}$ transitions, frequencies, and upper level energy and predicted line fluxes (in $\mathrm{erg} \mathrm{s}^{-1} \mathrm{~cm}^{-2}$ ) for the test case. The boldfaced transitions are used in the paper to illustrate how the line intensity varies with the parameters of the model (Figs. 2 to 9).

\begin{tabular}{|c|c|c|c|}
\hline Transition & $\begin{array}{l}\text { Frequency } \\
(\mathrm{GHz})\end{array}$ & $\begin{array}{c}E_{\mathrm{up}} \\
\left(\mathrm{cm}^{-1}\right)\end{array}$ & $\begin{array}{c}\text { Line flux } \\
\left(\mathrm{erg} \mathrm{s}^{-1} \mathrm{~cm}^{-2}\right)\end{array}$ \\
\hline $2_{1,2}-1_{1,1}$ & 140.8 & 15 & $5.5 \mathrm{E}-15$ \\
\hline $2_{1,1}-1_{1,0}$ & 150.4 & 16 & $5.5 \mathrm{E}-15$ \\
\hline $3_{1,3}-2,2$ & 211.2 & 22 & $1.5 \mathrm{E}-14$ \\
\hline $3_{1,2}-2_{1,1}$ & 225.6 & 23 & $1.4 \mathrm{E}-14$ \\
\hline $4_{1,4}-3,3$ & 281.5 & 32 & $2.4 \mathrm{E}-14$ \\
\hline $4_{3,2}-3_{3,1}$ & 291.3 & 98 & $1.2 \mathrm{E}-15$ \\
\hline $4_{3,1}-3_{3,0}$ & 291.3 & 98 & $1.2 \mathrm{E}-15$ \\
\hline $4_{1,3}-3_{1,2}$ & 300.8 & 33 & $1.8 \mathrm{E}-14$ \\
\hline $5_{1,5}-4_{1,4}$ & 351.7 & 43 & $2.4 \mathrm{E}-14$ \\
\hline $5_{3,3}-4_{3,2}$ & 364.2 & 110 & 2.6E-15 \\
\hline $5_{3,2}-4_{3,1}$ & 364.2 & 110 & $2.6 \mathrm{E}-15$ \\
\hline $5_{1,4}-4_{1,3}$ & 375.8 & 46 & $1.8 \mathrm{E}-14$ \\
\hline $6_{1,6}-5_{1,5}$ & 421.9 & 57 & $2.0 \mathrm{E}-14$ \\
\hline $6_{3,4}-5_{3,3}$ & 437.1 & 125 & $4.1 \mathrm{E}-15$ \\
\hline $6,3-5_{3,2}$ & 437.2 & 125 & $4.1 \mathrm{E}-15$ \\
\hline $6_{1,5}-5_{1,4}$ & 450.8 & 61 & $1.5 \mathrm{E}-14$ \\
\hline $7_{1,7}-6_{1,6}$ & 491.9 & 74 & $1.6 \mathrm{E}-14$ \\
\hline $7_{3,5}-6_{3,4}$ & 510.1 & 142 & $5.7 \mathrm{E}-15$ \\
\hline $7,4-6,3$ & 510.2 & 142 & $5.7 \mathrm{E}-15$ \\
\hline $7_{1,6}-6_{1,5}$ & 525.6 & 78 & $1.3 \mathrm{E}-14$ \\
\hline $8_{1,8}-7_{1,7}$ & 561.8 & 93 & $1.4 \mathrm{E}-14$ \\
\hline $8_{3,6}-7$, & 583.1 & 161 & $7.9 \mathrm{E}-15$ \\
\hline $8,5-73,4$ & 583.2 & 161 & $7.9 \mathrm{E}-15$ \\
\hline $8_{1,7}-7_{1,6}$ & 600.3 & 98 & $1.3 \mathrm{E}-14$ \\
\hline $9_{1,9}-8_{1,8}$ & 631.6 & 114 & $1.4 \mathrm{E}-14$ \\
\hline $9_{3,7}-8_{3,6}$ & 656.1 & 183 & $1.0 \mathrm{E}-14$ \\
\hline $9_{3,6}-8_{3,5}$ & 656.4 & 183 & $1.0 \mathrm{E}-14$ \\
\hline $9_{1,8}-8,7$ & 674.7 & 121 & $1.5 \mathrm{E}-14$ \\
\hline $10_{1,10}-9_{1,9}$ & 701.3 & 137 & $1.6 \mathrm{E}-14$ \\
\hline $10_{3,8}-9_{3,7}$ & 729.1 & 207 & $1.2 \mathrm{E}-14$ \\
\hline $10_{3,7}-9_{3,6}$ & 729.7 & 207 & $1.2 \mathrm{E}-14$ \\
\hline $10_{1,9}-9_{1,8}$ & 749.0 & 146 & $1.7 \mathrm{E}-14$ \\
\hline $11_{1,11}-10_{1,10}$ & 770.8 & 163 & $1.8 \mathrm{E}-14$ \\
\hline $11_{1,10}-10_{1,9}$ & 823.0 & 173 & $2.1 \mathrm{E}-14$ \\
\hline $12_{1,12}-11_{1,11}$ & 840.2 & 191 & $2.0 \mathrm{E}-14$ \\
\hline $12_{1,11}-11_{1,10}$ & 896.7 & 203 & $2.3 \mathrm{E}-14$ \\
\hline
\end{tabular}

$E_{\text {up }} \leq 80 \mathrm{~cm}^{-1}$ are relatively easily excited and become optically thick in this cold part, despite the low abundance of $3 \times 10^{-9}$. In contrast, the $\mathrm{H}_{2}^{13} \mathrm{CO}$ lines do not show this peak as the lines are optically thin and they therefore probe the inner regions of the envelope. Lines with $E_{\text {up }} \geq 80 \mathrm{~cm}^{-1}$ are not populated in the cold and less dense outer part of the envelope, and they probe the warmer and densest inner region, where $\mathrm{H}_{2} \mathrm{CO}$ is more abundant. In fact, the spectrum increases smoothly with the increase of the upper level energy of the transition and the two curves of the $\mathrm{H}_{2}^{12} \mathrm{CO}$ and $\mathrm{H}_{2}^{13} \mathrm{CO}$ lines run parallel at these level energies.

Figure 2 shows the contribution to the emission from different shells of the envelope for six transitions with different upper energy levels. The six lines in the plots have been chosen because they are observable with ground based telescopes and cover the $0-200 \mathrm{~cm}^{-1}$ range of the upper level

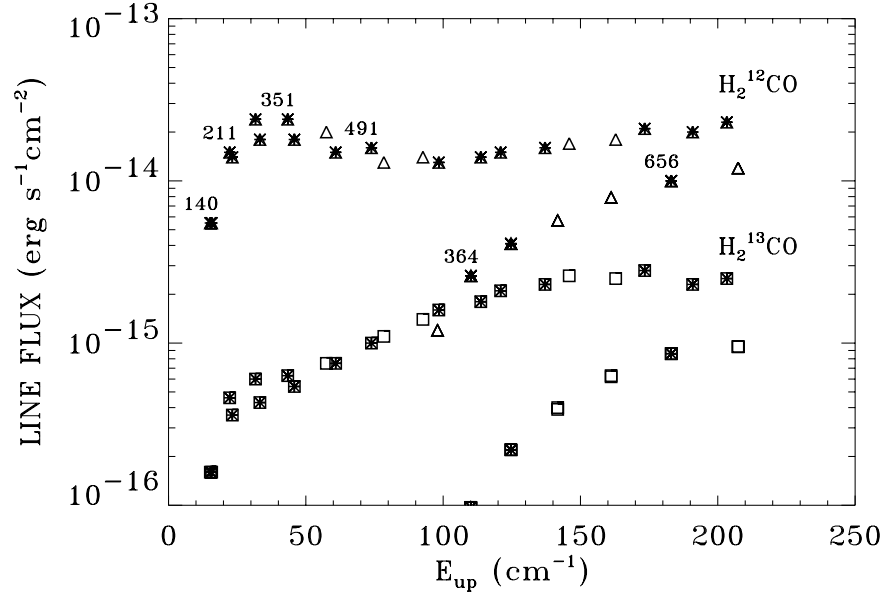

Fig. 1. Ortho $\mathrm{H}_{2} \mathrm{CO}$ line fluxes as function of the upper level energy of the transition. Triangles refer to $\mathrm{H}_{2}^{12} \mathrm{CO}$ transitions and squares to $\mathrm{H}_{2}^{13} \mathrm{CO}$ transitions; asterisks mark the transitions observable from ground-based telescopes; the six numbers associated with some triangles mark the frequencies (in $\mathrm{GHz}$ ) of the transitions whose emission as function of the radius is shown in Fig. 2. In each $\mathrm{H}_{2}^{12} \mathrm{CO}$ and $\mathrm{H}_{2}^{13} \mathrm{CO}$ line spectra, the series with $K_{-1}=1$ and $K_{-1}=3$ ladders are visible as those with the higher and lower fluxes respectively.
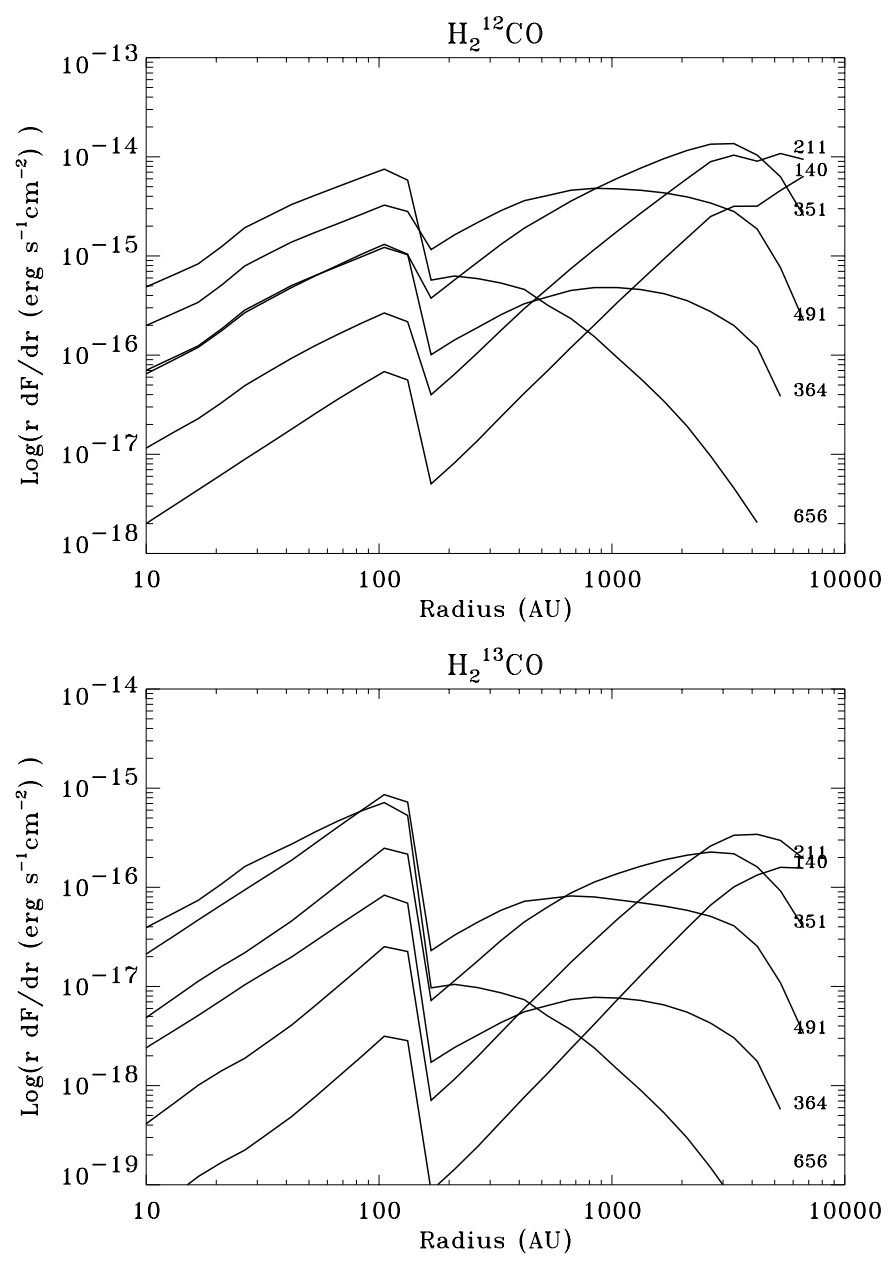

Fig. 2. Contribution to the line intensity as function of the distance from the central source, for six $\mathrm{H}_{2}^{12} \mathrm{CO}$ (upper panel) and the relevant $\mathrm{H}_{2}^{13} \mathrm{CO}$ (lower panel) transitions, whose $\mathrm{H}_{2}^{12} \mathrm{CO}$ frequencies in $\mathrm{GHz}$ are marked for each $\mathrm{H}_{2}^{12} \mathrm{CO}$ transition. 


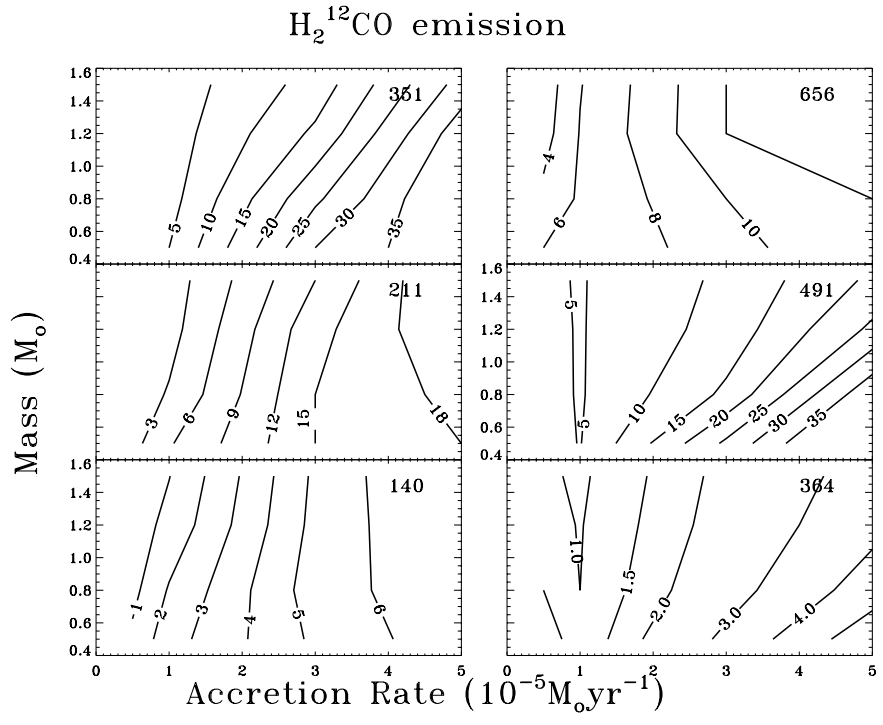

Fig. 3. Line intensity as function of the central mass and the mass accretion rate, for six $\mathrm{H}_{2}^{12} \mathrm{CO}$ transitions at 140, 211, 351, 364, 491 and $656 \mathrm{GHz}$ respectively. In these calculations line fluxes are in $10^{-15} \mathrm{erg} \mathrm{s}^{-1} \mathrm{~cm}^{-2}$. The $\mathrm{H}_{2} \mathrm{CO}$ abundance is $x_{\text {cold }}=3 \times 10^{-9}$ and $x_{\text {warm }}=3 \times 10^{-7}$.

energies. Specifically, the 492 and $656 \mathrm{GHz}$ transitions lie in a frequency range with the best atmospheric transmission in the $450-540 \mathrm{GHz}$ and $620-720 \mathrm{GHz}$ bands respectively, and hence are the easiest to observe at those frequencies. The six lines are used in the rest of the paper to illustrate how $\mathrm{H}_{2} \mathrm{CO}$ line intensities vary as function of the model parameters. Figure 2 illustrates that lines with higher upper level energies originate in the inner warm region, while lines with relatively lower upper level energies probe the outer, colder envelope. The relative contribution to the line intensity from the inner region ( $r \leq 150 \mathrm{AU})$ increases with increasing upper level energy of the transition. While the $\mathrm{H}_{2}^{12} \mathrm{CO}$ line intensities at 140, 211 and $351 \mathrm{GHz}$ are dominated by emission from the outer envelope, the line intensities at 364, 491 and $656 \mathrm{GHz}$ are dominated by the emission from the inner regions of the envelope. This behavior is even more marked for the $\mathrm{H}_{2}^{13} \mathrm{CO}$ transitions, which are optically thin and hence penetrate deeper into the envelope.

\subsection{Varying the parameters}

In this paragraph, we show how the $\mathrm{H}_{2} \mathrm{CO}$ line spectrum changes varying the four adjustable parameters of the model, namely the mass of the central object $M_{\star}$, the mass accretion rate $\dot{M}$, and the $\mathrm{H}_{2} \mathrm{CO}$ abundance in the outer and inner parts of the envelope, $x_{\text {cold }}$ and $x_{\text {warm }}$ respectively.

a) $M_{\star}$ and $\dot{M}$

Figure 3 shows the line intensities of the six $\mathrm{H}_{2}^{12} \mathrm{CO}$ lines for $M_{\star}$ varying between 0.4 and $1.6 M_{\odot}$ and $\dot{M}$ varying between 0.5 and $5 \times 10^{-5} M_{\odot} \mathrm{yr}^{-1}$. The 140 and $211 \mathrm{GHz}$ line intensity depend mostly on the accretion rate $\dot{M}$, and only weakly on the central mass $M_{\star}$, for the these relatively low lying lines originate in the outer part of the envelope, as pointed out above. In practice, the intensity of the 140 and $211 \mathrm{GHz}$ lines depends
$\mathrm{H}_{2}{ }^{12} \mathrm{CO} / \mathrm{H}_{2}{ }^{13} \mathrm{CO}$ RATIO

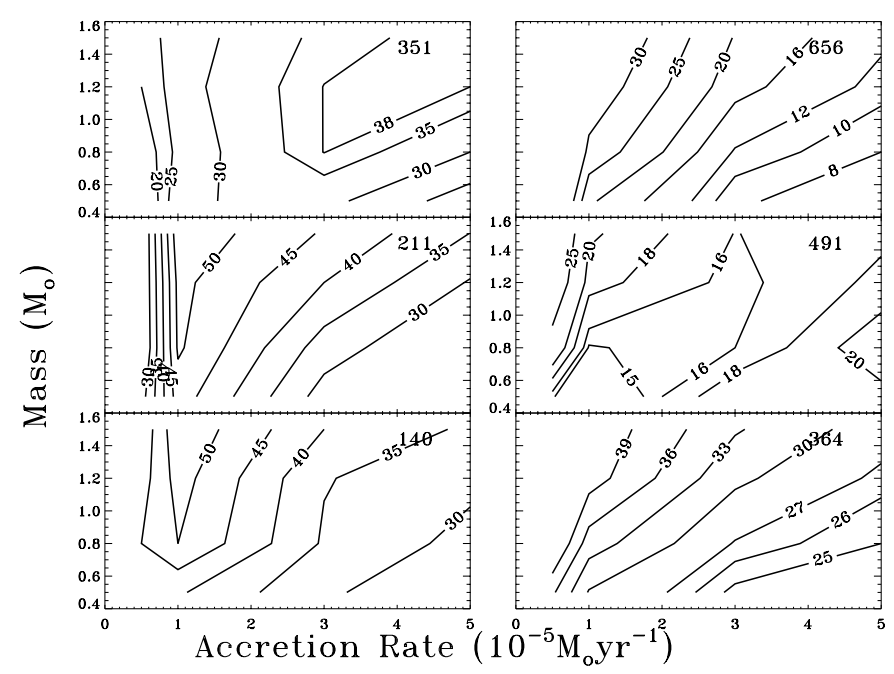

Fig. 4. The $\mathrm{H}_{2}^{12} \mathrm{CO}$ over $\mathrm{H}_{2}^{13} \mathrm{CO}$ line ratio of the same transitions of Fig. 3.

mainly on the column density of cold $\mathrm{H}_{2} \mathrm{CO}$, which is prevalently contained in the static part of the envelope. In the adopted inside-out framework, the static region depends on the mass accretion rate (Eqs. (1) and (4)). Higher upper level energy transitions have a larger contribution to the emission originating in the warm region, which is collapsing. Therefore, the line intensity depends, although weakly, also on the central mass, which sets the density and velocity of the collapsing region, in the inside-out framework (Eqs. (2) and (3)). Note that all six $\mathrm{H}_{2}^{12} \mathrm{CO}$ lines are optically thick, and hence the flux of the high lying lines is very sensitive to the size of the emitting region, e.g. where the grain desorption occurs. Figure 4 shows the $\mathrm{H}_{2}^{12} \mathrm{CO}$ over $\mathrm{H}_{2}^{13} \mathrm{CO}$ line ratio as function of $\dot{M}$ and $M_{\star}$. Note here that the transitions with a lower upper level energy also have lower $\mathrm{H}_{2}^{12} \mathrm{CO}$ over $\mathrm{H}_{2}^{13} \mathrm{CO}$ line ratios, both because of the different penetration of the lines and because of the physical structure of the envelope (see the discussion of Sect. 3.3). The plot shows that the $\mathrm{H}_{2}^{12} \mathrm{CO}$ over $\mathrm{H}_{2}^{13} \mathrm{CO}$ line ratio does not give a direct measure of the $\mathrm{H}_{2} \mathrm{CO}$ column density in the outer envelope, not even in the two lowest transitions (the 140 and $211 \mathrm{GHz}$ lines), because of the different penetration of the $\mathrm{H}_{2}^{12} \mathrm{CO}$ and $\mathrm{H}_{2}^{13} \mathrm{CO}$ lines, except for very low $\dot{M}$.

\section{b) $x_{\text {cold }}$ and $x_{\text {warm }}$}

Figure 5 shows the line intensities of the same six $\mathrm{H}_{2}^{12} \mathrm{CO}$ lines of Fig. 3, for $x_{\text {cold }}$ varying from $10^{-10}$ to $3 \times 10^{-8}$ and $x_{\text {warm }}$ varying from $10^{-8}$ to $3 \times 10^{-7}$. As already noted, the intensities of the lower level transitions are dominated by the emission from the cold part of the envelope and, therefore, they depend on $x_{\text {cold }}$. Actually, for a $0.8 M_{\odot}$ protostar accreting at $3 \times 10^{-5} M_{\odot} \mathrm{yr}^{-1}$, only the 656 and $364 \mathrm{GHz}$ lines are sensitive to $x_{\text {warm }}$ and only for relatively low $x_{\text {cold }}$ values. However, the $\mathrm{H}_{2}^{12} \mathrm{CO}$ over $\mathrm{H}_{2}^{13} \mathrm{CO}$ line ratios of the 364,491 and $656 \mathrm{GHz}$ lines are sensitive to $x_{\text {warm }}$, for the $\mathrm{H}_{2}^{13} \mathrm{CO}$ lines penetrate more into the inner parts of the envelope (Fig. 6). 


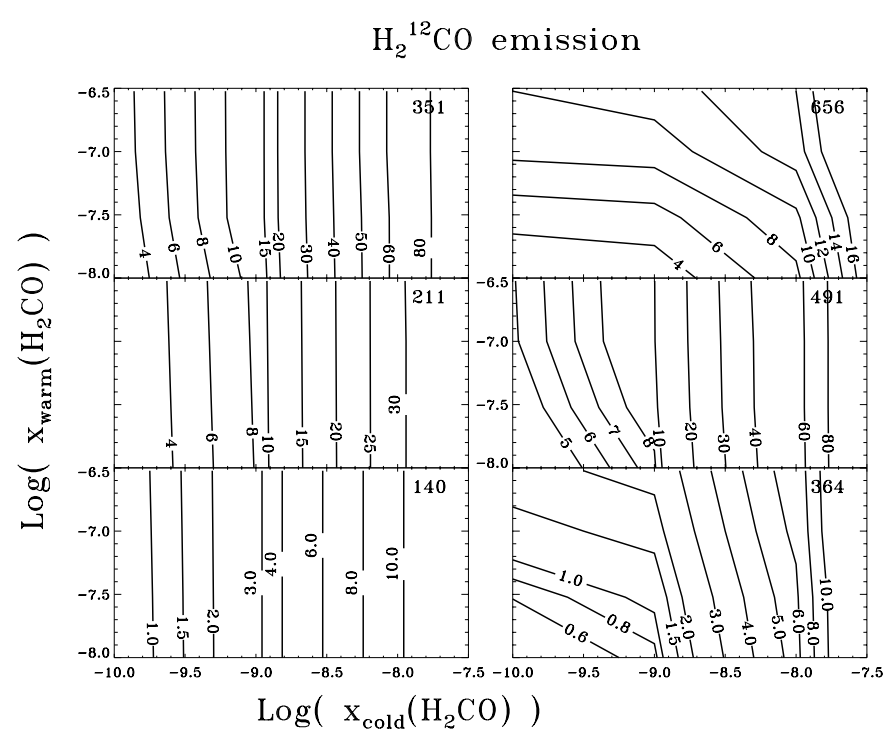

Fig. 5. Line intensity as function of the $\mathrm{H}_{2} \mathrm{CO}$ abundance in the outer and inner regions of the envelope, $x_{\text {cold }}$ and $x_{\text {warm }}$ respectively, for six $\mathrm{H}_{2}^{12} \mathrm{CO}$ transitions at $140,211,351,354,491$ and $656 \mathrm{GHz}$ respectively. Line fluxes are in $10^{-15} \mathrm{erg} \mathrm{s}^{-1} \mathrm{~cm}^{-2}$. In these calculations the mass of the central object is $0.8 M_{\odot}$ and the mass accretion rate is $3 \times 10^{-5} M_{\odot} \mathrm{yr}^{-1}$.

$$
\mathrm{H}_{2}{ }^{12} \mathrm{CO} / \mathrm{H}_{2}{ }^{13} \mathrm{CO} \text { RATIO }
$$

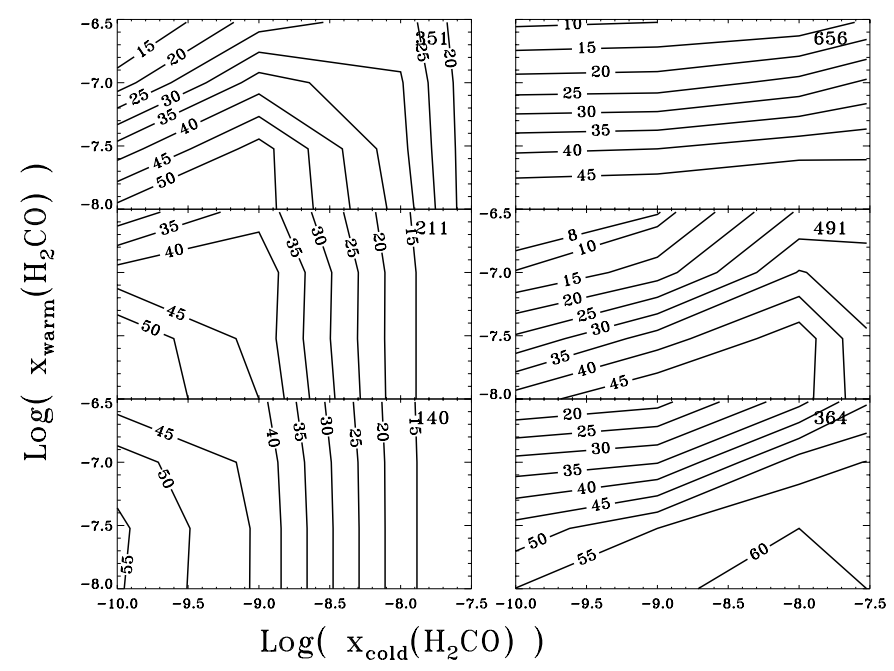

Fig. 6. The $\mathrm{H}_{2}^{12} \mathrm{CO}$ over $\mathrm{H}_{2}^{13} \mathrm{CO}$ line ratio of the same transitions of Fig. 5.

\subsection{Probing the collapse}

In this paragraph we discuss the possibility to use the $\mathrm{H}_{2} \mathrm{CO}$ lines to probe the collapse itself. Rather than modeling the line profile, we propose here to use the opacities and widths of several $\mathrm{H}_{2} \mathrm{CO}$ lines to probe that they originate in a centrally condensed envelope in which the velocity increases as the radius decreases, which is what happens in a collapsing envelope. Figure 7 shows the $\mathrm{H}_{2}^{13} \mathrm{CO} / \mathrm{H}_{2}^{12} \mathrm{CO}$ line intensity ratio multiplied by the $\left[{ }^{12} \mathrm{C}\right] /\left[{ }^{13} \mathrm{C}\right]$ ratio, equal to 70 (Boogert et al. 2000), in the case of a collapsing envelope and as

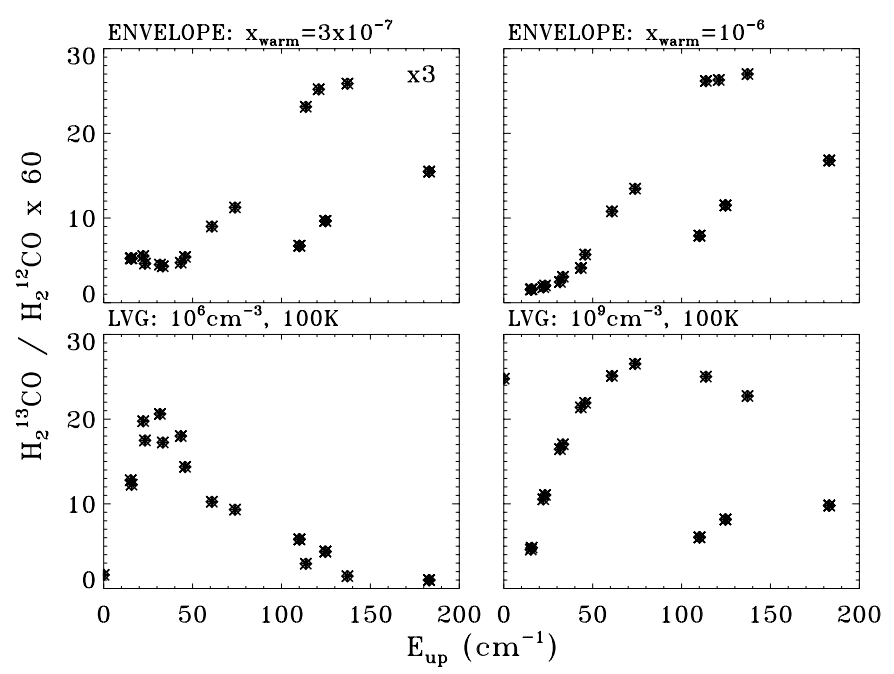

Fig. 7. $\mathrm{H}_{2}^{12} \mathrm{CO}$ over $\mathrm{H}_{2}^{13} \mathrm{CO}$ line ratio, multiplied by 60 , as function of the upper level energy in $\mathrm{cm}^{-1}$. The cases of emission from collapsing envelopes are reported, when $x_{\text {warm }}$ is equal to $3 \times 10^{-7}$ (upper left panel) and $1 \times 10^{-6}$ (upper right panel) respectively. Note that the $y$ axis is multiplied by 3 in the case of $x_{\text {warm }}=3 \times 10^{-7}$. The mass of the central object is $0.8 M_{\odot}, \dot{M}=3 \times 10^{-5}$, and $x_{\text {cold }}=3 \times 10^{-9}$ in these calculations. The lower panels show the $\mathrm{H}_{2}^{12} \mathrm{CO}$ over $\mathrm{H}_{2}^{13} \mathrm{CO}$ line ratio in the case of an isodense and isothermal slab of gas emitting at $100 \mathrm{~K}$. The left panel reports the case of a density equal to $10^{6} \mathrm{~cm}^{-3}$, i.e. subthermally populated levels, and the right panel the case of density equal to $10^{9} \mathrm{~cm}^{-3}$, i.e. LTE populated levels.

predicted by a non-LTE LVG code $^{1}$, which describes an isodense and isotherme slab with a velocity gradient. We deliberately compare the collapsing envelope model with a non-LTE LVG model because observations of $\mathrm{H}_{2} \mathrm{CO}$ lines, as those from other molecules, can be and have been interpreted as originating in shocks taking place very close to the central source, on scales comparable to the extent predicted by the collapsing model (e.g. see the discussion in Schöier et al. 2002). We want here to give a tool to distinguish the two cases observationally, based on the line opacity.

When the $\mathrm{H}_{2}^{13} \mathrm{CO}$ lines are optically thin and the $\mathrm{H}_{2}^{12} \mathrm{CO}$ lines are optically thick, and both $\mathrm{H}_{2}^{13} \mathrm{CO}$ and $\mathrm{H}_{2}^{12} \mathrm{CO}$ lines originate in the same region, the quantity in the plot of Fig. 7 represents the opacity $\tau_{12}$ of the $\mathrm{H}_{2}^{12} \mathrm{CO}$ lines. This is approximatively the case of the LVG approximation. However, in the case of the collapsing envelope, since the $\mathrm{H}_{2}^{13} \mathrm{CO}$ and $\mathrm{H}_{2}^{12} \mathrm{CO}$ lines do not originate in the same regions, as they have a different penetration depths, the plotted quantity is a combination of the different emitting area and opacity effects. Nevertheless, considering for a moment of the plotted quantity as the $\mathrm{H}_{2}^{12} \mathrm{CO}$ line opacity, it holds:

$\tau=\frac{A_{u \rightarrow l} \cdot c^{3}}{8 \pi v^{3}} \cdot \int n_{u}\left[\frac{n_{l} g_{u}}{n_{u} g_{l}}-1\right] \frac{\mathrm{d} z}{\delta v}$.

Since the line opacity in a centrally condensed envelope is local, i.e. lines are absorbed in the immediate vicinity of where they are emitted, $\tau$ is a measure of the density at which the line

1 The computational details of the non-LTE LVG code can be found in Ceccarelli et al. (2002). 
is emitted. The plot of Fig. 7 can now be interpreted: in a centrally condensed envelope, the opacity increases with increasing level energy, for higher lying lines originate in warmer and denser regions of the envelope, whereas in a isodense slab the opacity of the line has a peak corresponding roughly to the peak of the level population and roughly follows (depending on how much the levels are subthermally populated) the Boltzmann population law. The two distributions are definitively different, as Fig. 7 demonstrates, and can therefore be used to distinguish the case of a shock from a compact region close to the central source from the thermal emission of a collapsing envelope.

Figure 7 strictly proves that the emission originates in a centrally condensed region, but does not necessarily imply that the envelope is collapsing. However, a static envelope would give rise to lines with a relatively small linewidth due to the turbulence, whereas a collapsing envelope gives rise to lines whose linewidth increases with increasing upper level energy, as they probe inner regions with increasingly larger infall velocity. We conclude that the observation of increasing line opacity coupled with increasing linewidth as the energy of the line upper level increases provides a strong support in favor of a collapsing envelope. This method, which in principle is straigthforward, in practice can be difficult to apply, for the $\mathrm{H}_{2}^{13} \mathrm{CO}$ lines are weak for the current instrumentation, and for the possible simultaneous presence of an outflow component.

\section{Applications}

In this section we try to give some practical recipes to derive, in first approximation, the values of the parameters of the model from actual observations. However, we emphasize that the described method will give just some approximative estimate of the parameter values, and that a full modeling of the source is necessary to derive more precise estimates.

As a practical example, we will use the data obtained towards IRAS 16293-2422 (van Dishoeck et al. 1995; Loinard et al. 2000; Ceccarelli et al. 2001) and we will derive the approximate values of $M_{\star}, \dot{M}, x_{\text {cold }}$ and $x_{\text {warm }}$. We will then compare these results with those obtained with full modeling the $\mathrm{H}_{2} \mathrm{CO}$ emission from this source, as described in Ceccarelli et al. (2000b, 2001).

First, to facilitate the comparison, Table 3 reports the conversion factor of the line intensity in erg s${ }^{-1} \mathrm{~cm}^{-2}$ into the velocity integrated main beam temperature $T_{\mathrm{mb}} \Delta v$ observed at the IRAM and JCMT telescopes respectively, for the six lines of Fig. 3 (as well as the IRAS 16293-2422 data). Note that this is simply a conversion table, which does not take into account the convolution of the predicted emission with the telescope beam. This is strictly valid for a point-like source with respect to the telescope beam sizes. The reader should be aware that the convolution with the beam can introduce large factors of difference (by even a factor 5), if the emission originates in a region larger than that encompassed by the used beam, as it may be the case for low lying lines. In those cases, in order to use the plots of this article, the observer should take care to integrate the emission over the entire emitting region.

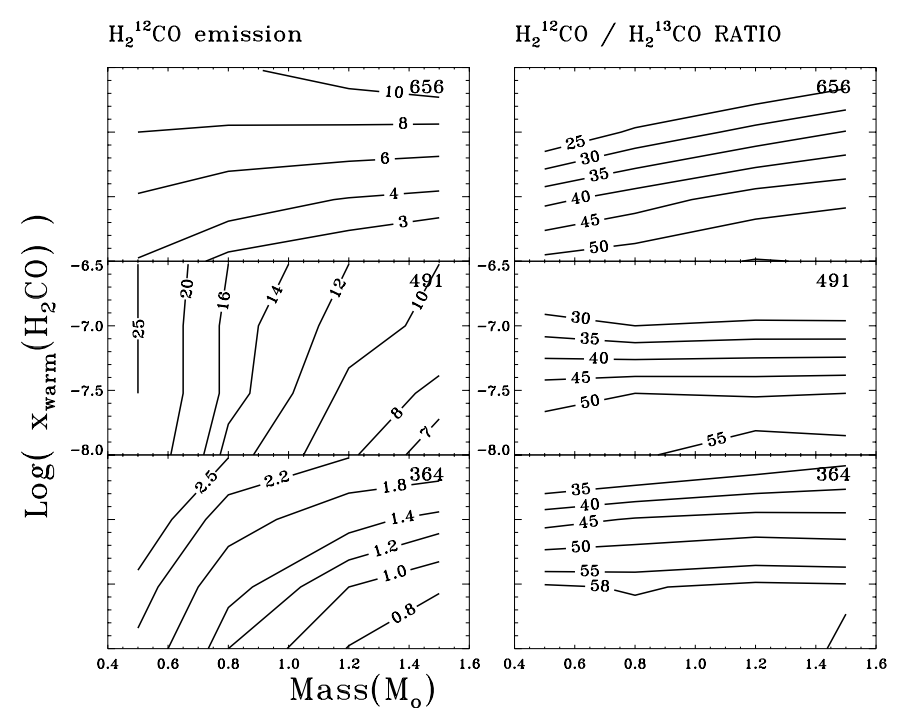

Fig. 8. Line intensity (left panels) and $\mathrm{H}_{2}^{12} \mathrm{CO}$ over $\mathrm{H}_{2}^{13} \mathrm{CO}$ line ratio (right panels) as function of the $\mathrm{H}_{2} \mathrm{CO}$ abundance in the inner regions of the envelope, $x_{\text {warm }}$, and the central mass, $M_{\star}$ for three $\mathrm{H}_{2}^{12} \mathrm{CO}$ transitions at 364, 491 and $656 \mathrm{GHz}$ respectively. Line fluxes are in $10^{-15} \mathrm{erg} \mathrm{s}^{-1} \mathrm{~cm}^{-2}$. In these calculations the outer $\mathrm{H}_{2} \mathrm{CO}$ abundance is $3 \times 10^{-9}$ and the mass accretion rate is $3 \times 10^{-5} M_{\odot} \mathrm{yr}^{-1}$.

\section{a) $M_{\star}$ and $x_{\text {warm }}$}

From the previous plots and discussion, it is now clear that the high lying transitions originate in the inner envelope: their intensities depend on the central mass $M_{\star}$ and the $\mathrm{H}_{2} \mathrm{CO}$ abundance in the inner envelope $x_{\text {warm. }}$. High lying lines can therefore be used to estimate these two parameters. Figure 8 shows the dependence of the 364,491 and $656 \mathrm{GHz}$ lines and their relative $\mathrm{H}_{2}^{12} \mathrm{CO}$ over $\mathrm{H}_{2}^{13} \mathrm{CO}$ line ratios, as function of $M_{\star}$ and $x_{\text {warm }}$. Note that these transitions are practically insensitive to $x_{\text {cold }}$ (Fig. 5) and very weakly sensitive to $\dot{M}$ (Fig. 3). Hence, the diagram of Fig. 8 can be used to constrain the values of $M_{\star}$ and $x_{\text {warm }}$. Comparison of the $364 \mathrm{GHz}$ data observed towards IRAS 16293-2422 with the plot of Fig. 8 gives the following values:

$M_{\star}=(0.6 \pm 0.2) M_{\odot}$ and $x_{\text {warm }} \leq 2 \times 10^{-7}$,

to compare with the values obtained with the full modeling of the source:

$M_{\star}=0.8 M_{\odot}$ and $x_{\text {warm }}=(1.1 \pm 0.4) \times 10^{-7}$. In the specific case of IRAS 16293-2422 the plot of Fig. 8 works hence reasonable well.

As a final remark, the plots of Fig. 8 can be used with single dish observations without the need of convolution, for the signal, originating in the warm part of the envelope, is likely to be encompassed by the telescope beam.

\section{b) $\dot{M}$ and $x_{\text {cold }}$}

Contrarely to the case discussed above, the low lying transitions originate in the outer envelope, and, hence, their intensities depend on both the mass accretion rate $\dot{M}$ and the $\mathrm{H}_{2} \mathrm{CO}$ abundance in the outer envelope $x_{\text {cold }}$. Figure 9 shows the dependence of three $\mathrm{H}_{2}^{12} \mathrm{CO}$ lines at 140,211 , and $351 \mathrm{GHz}$, as function of $\dot{M}$ and $x_{\text {cold }}$. As already cautioned, to use the plots of Fig. 9 one needs to compare the observed flux integrated over the entire envelope. Using the data in Table 3, we derive 
Table 3. Conversion factors of the line intensity in $\mathrm{erg} \mathrm{s}^{-1} \mathrm{~cm}^{-2}$ into the velocity integrated main beam temperature $T_{\mathrm{mb}} \Delta v$ observed at the IRAM and JCMT telescopes respectively, for the six lines of Fig. 3. Last column quotes the $T_{\mathrm{mb}} \Delta v$ observed towards IRAS 16293-2422 (van Dishoeck et al. 1995; Loinard et al. 2000; Ceccarelli et al. 2001)..$^{a}$ Line intensity integrated over the envelope, which extends up to $20^{\prime}$ in radius; ${ }^{b}$ the value 27 is that measured on the central beam, hence the value over the entire envelope is larger than this.

\begin{tabular}{|c|c|c|c|c|c|c|}
\hline \multirow[t]{2}{*}{ Transition } & \multirow{2}{*}{$\begin{array}{c}\text { Frequency } \\
(\mathrm{GHz})\end{array}$} & \multirow{2}{*}{$\begin{array}{c}\text { IRAM } T_{\mathrm{mb}} \Delta v \\
\left(\mathrm{~K} \mathrm{~km} \mathrm{~s}^{-1}\right)\end{array}$} & \multirow{2}{*}{$\begin{array}{c}\text { JCMT } T_{\mathrm{mb}} \Delta v \\
\left(\mathrm{~K} \mathrm{~km} \mathrm{~s}^{-1}\right)\end{array}$} & \multirow{2}{*}{$\begin{array}{l}\text { Line flux } \\
\mathrm{erg} \mathrm{s}^{-1} \mathrm{~cm}^{-2}\end{array}$} & \multicolumn{2}{|c|}{ IRAS 16293-2422 } \\
\hline & & & & & $\left(\mathrm{K} \mathrm{km} \mathrm{s}^{-1}\right)$ & $\mathrm{H}_{2}^{12} \mathrm{CO} / \mathrm{H}_{2}^{13} \mathrm{CO}$ \\
\hline $22_{1,2}-1_{1,1}$ & 140 & 1 & & $2.4 \mathrm{E}-17$ & 16.6 & 29 \\
\hline $3_{1,3}-2,1$ & 211 & 1 & & $3.8 \mathrm{E}-17$ & $411^{a}$ & $\geq 27^{b}$ \\
\hline $5_{1,5}-4_{1,4}$ & 351 & & 1 & $2.2 \mathrm{E}-16$ & 38.3 & 28 \\
\hline $5_{3,3}-4_{3,2}$ & 364 & & 1 & $2.3 \mathrm{E}-16$ & 11.9 & $\geq 40$ \\
\hline $7_{1,7}-6_{1,6}$ & 491 & & 1 & $4.2 \mathrm{E}-16$ & & \\
\hline $9_{3,7}-8_{3,6}$ & 656 & & 1 & $5.2 \mathrm{E}-16$ & & \\
\hline
\end{tabular}

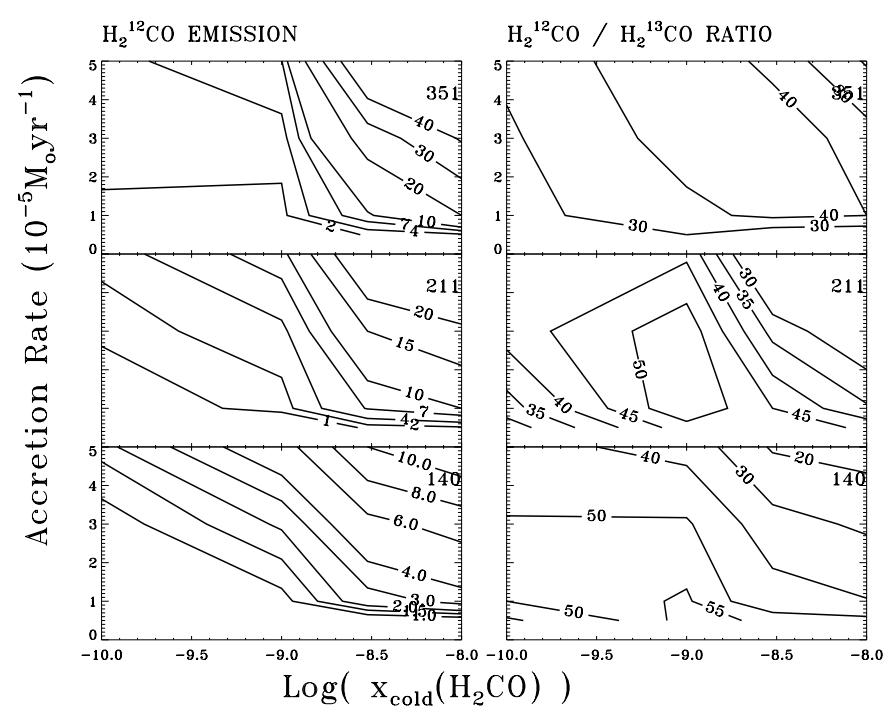

Fig. 9. Line intensity (left panels) and $\mathrm{H}_{2}^{12} \mathrm{CO}$ over $\mathrm{H}_{2}^{13} \mathrm{CO}$ line ratio (right panels) as function of the $\mathrm{H}_{2} \mathrm{CO}$ abundance in the outer regions of the envelope, $x_{\text {cold }}$, and as function of the mass accretion rate, $\dot{M}$, for three $\mathrm{H}_{2}^{12} \mathrm{CO}$ transitions at 140,211 , and $351 \mathrm{GHz}$ respectively. Line fluxes are in $10^{-15} \mathrm{erg} \mathrm{s}^{-1} \mathrm{~cm}^{-2}$. In these calculations, the mass of the central object is $0.8 M_{\odot}$ and the $\mathrm{H}_{2} \mathrm{CO}$ abundance in the warm regions of the envelope, $x_{\text {warm }}$, is $1 \times 10^{-7}$.

the following approximate values for IRAS 16293-2422:

$\dot{M} \sim 3 \times 10^{-5}$ and $x_{\text {cold }} \sim 3 \times 10^{-9}$

against the values obtained with the full modeling:

$\dot{M} \sim 3.5 \times 10^{-5} M_{\odot} \mathrm{yr}^{-1}$ and $x_{\text {cold }}=(1.1 \pm 0.3) \times 10^{-9}$.

Also in this case the agreement is reasonable.

\section{The web site MEPEW}

Tables with the computed line fluxes used to derive the plots shown in the previous section can be found in the web site www-laog.obs.ujf-grenoble.fr/ ceccarel/ mepew/mepew.html. Results of the model for sources whose luminosities are equal to $1,2,5,10$ and $15 L_{\odot}$ are also reported on this web site. The latter calculations have been obtained to specifically analyze the sources studied in Maret et al. (in preparation), and the free parameters are in those cases the $\mathrm{H}_{2} \mathrm{CO}$ abundance in the inner and outer parts of the envelope. The site contains tables with the list of the predicted fluxes of the formaldehyde transitions, with frequencies between $140 \mathrm{GHz}$ and $900 \mathrm{GHz}$. Other complementary informations, such as the line opacity and the peak of the radial distribution of the line emission, are also quoted in the tables. For all sources, results are tabulated for formaldehyde abundances $x_{\text {cold }}$ varying from $10^{-12}$ to $10^{-9}$, and $x_{\text {warm }}$ varying from $10^{-10}$ to $10^{-4}$ respectively. Further instructions for the retrieval of the tables as well as their format can be found in the web site. We think that this information may be useful for estimates of the formaldehyde abundance in protostellar envelopes by other authors, who can easily compare their observations with theoretical predictions.

Aiming to give a tool also for first order computations, the site hosts tables with predictions of a non-LTE LVG model, whose computational details are given in Ceccarelli et al. (2002). Several molecules are reported and more will be added in the future. Details on the relevant data used for each molecule are given in the site. Flux predictions are given for a large range of temperatures (typically from 10 to $1000 \mathrm{~K}$ ) and densities (typically from $10^{3}$ to $10^{9} \mathrm{~cm}^{-3}$ ), as well as column densities. Specifically, the column densities are such that the lines are from optically thin to heavy optically thick (typically $\tau \sim 10$ ).

\section{Conclusions}

We reported theoretical predictions of the formaldehyde line emission from the envelopes of low mass protostars. We have shown that the simultaneous observations of appropriately selected transitions permit to approximatively constrain the mass accretion rate, the mass of the central mass and the formaldehyde abundance in the outer cold and inner warm parts of the envelope. We have also shown that the formaldehyde line spectrum of an infalling envelope differs greatly from that of an homogeneous slab, which can be a rough representation of the shocked gas at the interface of the outflowing gas with the surroundings. In principle, hence, the observed formaldehyde spectrum can distinguish between the two cases (other than, obviously, the profile, when it is resolved and the linewidth is very large - which, unfortunately, may not be always the case).

The capabilities to observe molecular transitions have largely improved in the last years and will further improve in the next years with the advent of planned new submillimeter telescopes (APEX, HERSCHEL, ALMA). Interpretation of multifrequency observations require detailed modeling of the 
line emission, as that reported in this article, or in other articles (see e.g. the Introduction). For this reason, we have posted the results for sources with different luminosities on the web site www-laog.obs.ujf.grenoble.fr/ ceccarel/ mepew/mepew.html, to provide a fast tool to the interested observers. The site provides also calculations obtained with a non-LTE LVG code for several molecules, for first order estimates of the average density and temperature of the emitting gas.

Acknowledgements. We wish to thank the referee, Dr F.L. Schöier, for carefully reading the manuscript and his useful comments.

\section{References}

Adelson, L. M., \& Leung, C. M. 1988, MNRAS, 235, 349

Boogert, A. C. A., Ehrenfreund, P., Gerakines, P. A., et al. 2000, A\&A, 353, 349

Cazaux, S., Tielens, A. G. G. M., Ceccarelli, C., et al. 2003, ApJ, 593, L51

Ceccarelli, C., Hollenbach, D. J., \& Tielens, A. G. G. M. 1996, ApJ, 471, 400 (CHT96)

Ceccarelli, C., Castets, A., Caux, E., et al. 2000a, A\&A, 355, 1129

Ceccarelli, C., Loinard, L., Castets, A., Tielens, A. G. G. M., \& Caux, E. 2000b, A\&A, 357, L9

Ceccarelli, C., Loinard, L., Castets, A., et al. 2001, A\&A, 372, 998

Ceccarelli, C., Baluteau, J.-P., Walmsley, M., et al. 2002, A\&A, 383, 603

Choi, M. 2001, ApJ, 553, 219

Choi, M. 2002, ApJ, 575, 900

Di Francesco, J., Myers, P., Wilner, D., Ohashi, N., \& Mardones, D. 2001, ApJ, 562, 770

Evans, N. J. 1999, ARA\&A, 37, 311
Gibb, E. L., Whittet, D. C. B., Schutte, W. A., et al. 2000, ApJ, 536, 347

Green, S. 1991, ApJ, 76, 979

Gregersen, E. M., Evans, II N. J., Zhou, S., \& Choi, M. 1997, ApJ, 484, 256

Leung, C. M., \& Brown, R. L. 1977, ApJ, 214, L73

Loinard, L., Castets, A., Ceccarelli, C. et al. 2000, A\&A, 359, 1169

Mangum, J., Wootten, A., Wadiak, E., \& Loren, R. B. 1990, ApJ, 348, 542

Mangum, J., \& Wootten, A. 1993, ApJS, 89, 123

Mangum, J., Wootten, A., \& Barsony, M. 1999, ApJ, 526, 845

Mardones, D., Myers, P. C., Tafalla, M., et al. 1997, ApJ, 489, 719

Maret, S., Ceccarelli, C., Caux, E., Tielens, A. G. G. M., \& Castets, A. $2002 a, A \& A, 395,573$

Narayanan, G., \& Walker, C. K. 1998, ApJ, 508, 780

Narayanan, G., Walker, C. K. \& Buckley, H. D. 1998, ApJ, 496, 292

Schöier, F. L., Jørgensen, J. K., van Dishoeck, E. F., \& Blake, G. A. 2002, A\&A, 390, 1001

Shu, F. H. 1977, ApJ 214, 488

Tielens, A. G. G. M, \& Allamandola, L. 1987, in Interstellar Processes, ed. D. J. Hollenbach, H. A. \& Thronson (Reidel Publishing Company), 397

van Dishoeck, E. F., Blake, G. A., Jansen, D. J., \& Groesbeck T. D. 1995, ApJ, 447, 760

van Dishoeck, E. F., \& Blake, J. 1998, ARA\&A, 36, 317

van der Tak, F. S., van Dishoeck, E. F., \& Caselli, P. 2000, A\&A, 361, 327

Walker, C. K., Lada, C. J., Young, E. T., Maloney, P. R., \& Wilking, B. A. 1986, ApJ, 309, L47

Walker, C. K., Narayanan, G., \& Boss, A. P. 1994, ApJ, 431, 767

Williams, J. P., \& Myers, P. C. 1999, ApJ, 511, 208

Wilson, T. L., \& Rood, R. T. 1994, ARA\&A, 32, 191

Zhou, S. 1992, ApJ, 394, 204

Zhou, S. 1995, ApJ, 442, 685 